\title{
Dynamical test of interaction potentials for colloidal suspensions
}

\author{
F. Bitzer, T. Palberg, ${ }^{*}$ H. Löwen, ${ }^{*}{ }^{\dagger}$ R. Simon, and P. Leiderer \\ Universität Konstanz, Fakultät für Physik, D-78434 Konstanz, Germany
}

(Received 10 January 1994)

\begin{abstract}
An analytical formula for the ratio between the long- and short-time self-diffusion coefficient $D_{L} / D_{S}$ is presented as a result of extensive Brownian dynamics simulations for Yukawa systems. In addition, forced Rayleigh scattering experiments are performed to determine $D_{L} / D_{S}$ for suspensions of highly charged latex spheres with a variable low volume fraction immersed in electrolytes of variable concentration. Experimental parameters were precisely controlled using advanced preparational methods. Comparing experimental data to the analytical formula we perform a sensitive dynamical test of Yukawa models for the interaction potential. The observed quantitative agreement provides strong evidence for the validity of the renormalization procedures suggested within the Poisson-Boltzmann cell model [J. Chem. Phys. 80, 5776 (1984)]. Deviations observed for the Dejarguin-Landau-Verwey-Overbeek potential and other models are discussed.
\end{abstract}

PACS number(s): 82.70.Dd, 66.10.-x

\section{INTRODUCTION}

Transport processes in charge stabilized colloidal suspensions are of major importance both in biological systems and in industrial processing. Diffusional [1], rheological [2], and electrokinetic [3] properties have been studied extensively in systems of so called monodisperse particles which may be synthesized with well-defined particle dimensions and surface chemistry. The effective interaction between the colloidal particles can be varied experimentally by changing the packing fraction $\Phi$, the density of screening electrolyte $\rho_{S}$, or the particle charge $Z$. Highly charged colloidal spheres show pronounced ordering even at extremely low packing fractions, if $\rho_{S}$ is kept sufficiently low; whereas sterically stabilized hard spheres show a freezing transition at $\Phi \geq 0.494$. Changes in the fundamental processes of diffusional transport due to the formation of cages of neighboring particles are observable in such model suspensions with high accuracy by light scattering techniques.

In the presence of fluid order the time dependent mean squared displacement $\left\langle r^{2}\right\rangle$ of a colloidal sphere of radius $a$ in a medium of viscosity $\eta$ shows two linear regimes. At short times the particle diffuses quasi freely. The selfdiffusion coefficient for short times $t D_{S}=\left\langle r^{2}\right\rangle / 6 t$ takes the Stokes-Einstein value $D_{0}=k_{B} T / 6 \pi \eta a$, where $k_{B}$ is the Boltzmann constant and $T$ the temperature [4]. Corrections to this value may consider hydrodynamic interaction at elevated volume fractions $\Phi>0.01$ [5] or electrolyte friction at typically millimolar salt concentrations [6]. Any direct repulsive interaction with other particles considerably reduces the diffusional transport. The structural relaxation of the surrounding cage of neighbor-

\footnotetext{
*Corresponding authors.

†Permanent address: Sektion Physik, Ludwigs-Maximilians Universität, D-80333 München, Germany.
}

ing particles then becomes the limiting process of particle diffusion. At longer times, after several rearrangements of the cage, a second linear regime is reached. The longtime self-diffusion coefficient $D_{L}$ is found to decrease with increasing strength of interaction. This has been shown experimentally for both charge and sterically stabilized particles by means of different techniques, including dynamic light scattering (DLS) [7,8], forced Rayleigh scattering (FRS) [9-11] and fluorescence recovery after photobleaching [12]. Most of the experimental work so far was confined to hard sphere systems. Data on $D_{L}$ in the fluid phase of charge stabilized systems are rare and mostly consider a variation of only one experimental condition ( $\Phi$ or $\rho_{S}$ ).

It is convenient to express the decrease of $D_{L}$ in terms of the self-diffusion ratio $D_{L} / D_{S}$, which is one for a noninteracting system. It has recently been shown that this ratio has a universal value of $D_{L} / D_{S}=0.098$ at the freezing transition of colloidal fluids [10]. This constitutes a dynamical freezing criterion for Brownian systems of arbitrary interaction potential.

On the theoretical side, results of Brownian dynamics (BD) simulations have been compared to analytical predictions on $D_{L}$. The theoretical description of the longtime self-diffusion behavior is based on the memory function formalism. Kineticlike theories using both two particle dynamics and the exact short-time behavior of the friction kernel give the best agreement with BD simulation results [13]. Nevertheless the predicted values for $D_{L}$ are still off by some $30 \%$ from the simulation results.

In this paper we present extensive data for $D_{L} / D_{S}$ from both BD simulations and forced Rayleigh scattering experiments. The experiments were performed on monodisperse charge-stabilized latex spheres of known true surface charge and precisely controlled particle density and salt concentration. A comparison between the data sets of the experiments and BD simulations gives direct insight into the particle interaction. In particular, a number of different theoretical descriptions have been pro- 
posed, where the particle interaction is described in terms of a Yukawa pair potential. The Yukawa parameters, however, differ for the different theories. In this paper we will use a dynamical quantity, namely, the ratio of the self-diffusion coefficients $D_{L} / D_{S}$ to discriminate between different Yukawa models.

Usually a comparison of theoretically derived to experimentally determined fluid static structure factors $S(k)$ is used to judge the validity of interaction models. The problem then is that $S(k)$ is rather insensitive with respect to details of the particle interaction. In most cases $S(k)$ keeps the same shape and it is only the amplitude responding to the average strength of interaction. On the other hand, dynamical quantities depend much more sensitively on the interaction and thus provide a more stringent test for the interaction potential, respectively, the Yukawa parameters. This was demonstrated in the molecular dynamics of Yukawa systems by Robbins, Kremer, and Grest [14]. They have shown that two different Yukawa models may lead to practically identical static pair correlations but qualitatively different dynamical quantities, i.e., velocity autocorrelation functions. This sensitivity of dynamical quantities with respect to the details of the particle interaction is here used to discriminate between different Yukawa models by comparing the experimental data of particle self-diffusion to data from computer simulation.

A similar comparison was recently performed by $\mathrm{Härtl}$ et al. [7]. Our work, however, differs from theirs in two major aspects. First, our computer simulation is more extensive, scanning the whole fluid regime of the twodimensional Yukawa phase diagram and providing an explicit formula for $D_{L} / D_{S}$ which facilitates a direct comparison with different Yukawa models. Second, the true surface charge of our experimental samples is known. In contrast to Ref. [7], where the surface charge was obtained by fitting $S(k)$, here there is no fit parameter in our comparison. A quantitative agreement between theory and experiment thus provides strong evidence for the validity of the theoretical model. Consequently the idea of a dynamical test for interaction potential could not be pursued in Ref. [7] or other papers.

Our paper is organized as follows: After a brief description of the simulation procedures and the experimental method we present a detailed comparison between the results obtained. We finally discuss the impact of the found quantitative agreement for further developments of theoretical descriptions.

\section{BROWNIAN DYNAMICS SIMULATIONS OF YUKAWA SYSTEMS}

In this chapter we describe the results of extensive Brownian dynamics (BD) simulations for the long time self-diffusion coefficient $D_{L}$. Hydrodynamic interactions are ignored. The BD algorithm is explained in detail in Refs. [12,15]. The short-time diffusion coefficient $D_{S}$ enters in a natural way into the BD simulations and determines the time scale. We are considering systems of particles interacting via a pairwise Yukawa potential:

$$
V(r)=U \frac{e^{-\kappa r}}{r},
$$

frequently used to describe the effective interaction between charged colloidal particles. In (1) $1 / \kappa$ sets the length scale and $U$ the energy scale. By means of a suitable scaling $[14,16]$ for a given particle number density $\rho$ and temperature $T$, a Yukawa system can be characterized by two-dimensionless parameters:

$$
\lambda=\kappa\left(\frac{1}{\rho}\right)^{1 / 3}
$$

and

$$
\widetilde{T}=\frac{k_{B} T}{U} \lambda e^{\lambda} .
$$

Recently, the phase diagram of a Yukawa system as a function of $\widetilde{T}$ and $\lambda$, was calculated via computer simulation by Meijer and Frenkel [16]. Their range of $3<\lambda<7$ coincides with the range of $\lambda$ under consideration in this work. Interpolating their data by a polynomial fit one finds the following analytical expression for the freezing line $\widetilde{T}_{f}(\lambda)$ in the $(\widetilde{T}, \lambda)$ plane $[18]$ :

$$
\begin{aligned}
\widetilde{T}_{f}(\lambda)= & 0.009+0.0303 \lambda-0.00997 \lambda^{2}+0.0035 \lambda^{3} \\
& -0.000245 \lambda^{4}
\end{aligned}
$$

See also Stevens and Robbins [17], who report a higher melting temperature at large $\lambda$.

We have performed a series of 40 different BD runs to calculate the self-diffusion ratio $D_{L} / D_{S}$ scanning the whole fluid regime in the $(\widetilde{T}, \lambda)$ phase diagram for $\widetilde{T} \geq \widetilde{T}_{f}$ and $\lambda \leq 7$. In Refs. [10] and [13] only a few discrete points in the $(\widetilde{T}, \lambda)$ diagram were taken. On the freezing line $D_{L} / D_{S}$ was previously found to have the universal value $D_{L} / D_{S}=0.098$ [10]. In order to find a simple analytical expression for $D_{L} / D_{S}$ in the fluid regime we have used this fact and tried an exponential ansatz for the scaled inverse temperature. We found the fit:

$$
\frac{D_{S}}{D_{L}}=0.6286 \exp \left[-1.8585 \frac{\widetilde{T}_{f}(\lambda)}{\widetilde{T}}\right] \text {. }
$$

This Arrhenius-like fit was also used to fit the longtime self-diffusion coefficients from molecular dynamics data of Yukawa systems [18]. Equation (5) is a good fit with a relative error of less than $3 \%$ in $D_{L} / D_{S}$, if $D_{L} / D_{S} \leq 0.6$, i.e., at strong particle interaction. We emphasize, however, that the Arrhenius-like behavior results simply from the fit procedure, but does not necessarily imply a physical background of a hidden activated dynamical process.

A more general fit working for arbitrary $D_{L} / D_{S}$ with an error of less than $4 \%$ is provided by the formula:

$$
D_{L} / D_{S}=A_{1} e^{--\mu_{1} t}+A_{2} e^{\left.-i \mu_{2}+\mu_{3} \lambda\right) t}+A_{3} \text {, }
$$

with $t=\widetilde{T}_{f}(\lambda) / \widetilde{T}$ and the constants $A_{1}=0.646$, $A_{2}=0.318, A_{3}=0.0360, \mu_{1}=2.343, \mu_{2}=31.702$, and $\mu_{3}=6.053$. Again the double Arrhenius-like fit is a convenient analytical form but does not imply a physical 
background of thermally activated diffusion processes. Note that in both formulas the dynamical freezing criterion $D_{L} / D_{S}=0.098$ for $t=1$ and the high temperature or low density limit of $D_{L} / D_{S}=1$ for $t \rightarrow \infty$ are included.

Equation (6) provides a simple but general result for the self-diffusion ratio $D_{L} / D_{S}$ in Brownian Yukawa fluids. A comparison with experimental data then facilitates discrimination between different theoretical $\mathrm{Yu}$ kawa models used to describe the interaction between charged colloidal spheres.

\section{FORCED RAYLEIGH SCATTERING EXPERIMENTS}

The particles used here were well characterized Polystyrene Latex spheres of hydrodynamic radius $a=51 \mathrm{~nm}$ (dynamic light scattering) and a number of titrated sulfate surface groups of $N=1200$ (Lot 2010M9R, Seradyn, U.S.A.). Sample preparations used a recently reported advanced deionization technique [19]. Salt concentrations are controlled via conductometric measurements taking into account the conductivity of the suspending medium. The experimental parameters were adjusted with a residual uncertainty of $1 \%$ in the volume fraction $\Phi$ (using static light scattering) and $2 \%$ in the concentration of excess electrolyte $c_{S}$. At typical experimental conditions of both low volume fraction and low salt concentration, the titration of interaction dependent quantities, yields a true surface charge number of $Z=580$ (resulting from incomplete dissociation of surface groups) [20].

To determine the self-diffusion coefficients forced Rayleigh scattering (FRS) was used [9,11]. A small fraction of the particles is uv-sensitized with 6-nitro-BIPS (Merck, Germany). An absorption grid of variable wavelength is formed in the sample by two crossed uv-laser beams. Its diffusional decay is monitored via the Bragg reflection of a He-Ne laser beam. The monoexponential signal is evaluated for the self-diffusion coefficient, using independently measured corrections for the incoherent and coherent scattering backgrounds and the relaxation time of the dye.

Up to 20 single measurements are averaged in a run (statistical error $\Delta D \leq 1 \%$ ) and typically three runs are taken at each set of experimental conditions. The residual uncertainties of typically less than $3 \%$ in $D_{L}\left(c_{S}, \Phi, Z\right)$ are mainly due to the remaining variations in the experimental parameters.

For noninteracting systems [static structure factor $S(k)=1]$ short- and long-time self-diffusion coefficients coincide: $D_{S}=D_{L}$. Due to electrolyte friction at typically millimolar salt concentration $(\kappa a \approx 1)[6]$ the measured short-time diffusion coefficient takes values slightly below the Stokes-Einstein value measured at large $c_{S}$ : $D_{S} \leq D_{0}=k_{B} T / 6 \pi \eta a$ (where $\eta$ is the viscosity of the suspending medium). In the following we only compare the ratio $D_{L} / D_{S}$, which is unaffected by electrolyte friction.

\section{RESULTS}

In comparing the experimental data with the theoretical fit formula for a Yukawa interaction, three different theoretical models and an empirically found relation for the Yukawa parameters are considered. The repulsive part of the Dejarguin-Landau-Verwey-Overbeek (DLVO) potential uses the solution of the linearized PoissonBoltzmann (PB) equation. It also includes a correction for the finite particle radius. The number of dissociated surface groups $Z$ and the bulk values of the total small ion concentration are used as input for $U$ and $\kappa$ :

$$
U_{\mathrm{DLVO}}=\frac{Z^{2} e^{2}}{4 \pi \varepsilon_{0} \varepsilon}\left(\frac{\exp \left(\kappa_{\mathrm{DLVO}} a\right)}{\left(1+\kappa_{\mathrm{DLVO}} a\right)}\right)^{2},
$$

with the Debye-Hückel expression:

$$
\kappa_{\mathrm{DLVO}}^{2}=e^{2}\left(2 \rho_{S}+\rho Z\right) / \varepsilon \varepsilon_{0} k_{B} T,
$$

where $e$ is the elementary charge, $\varepsilon \varepsilon_{0}$ is the dielectric permittivity of the suspending medium, $\rho_{S}=1000 N_{A} c_{S}$ is the number density of monovalent salt ions.

A second approach is to solve the mean sphèrical approximation (MSA) analytically in the framework of the primitive model for both macroions and counterions. This leads to an effective interparticle interaction which has the Yukawa form (1) with the same $\kappa$ as in the DLVO expression but an increased effective charge $Z_{\mathrm{MSA}}>\boldsymbol{Z}$. Explicitly one gets [21]

$$
U_{\mathrm{MSA}}=\frac{Z_{\mathrm{MSA}}^{2} e^{2}}{4 \pi \varepsilon_{0} \varepsilon}\left(\frac{\exp \left(\kappa_{\mathrm{MSA}} a\right)}{\left(1+\kappa_{\mathrm{MSA}} a\right)}\right)^{2}
$$

and

$$
\kappa_{\mathrm{MSA}}^{2}=e^{2}\left(2 \rho_{S}+\rho Z\right) / \varepsilon \varepsilon_{0} k_{B} T .
$$

The renormalization procedures $[22,23]$ use a Yukawa fit to the numerical solution of the nonlinearized PB equation within a spherical Wigner-Seitz cell. This results in a third version for a Yukawa interaction which is called the Poisson-Boltzmann cell model (PBC). The simple analytical form (1) is retained for somewhat smaller renormalized charge numbers $Z_{\mathrm{PBC}}<Z$ :

$$
U_{\mathrm{PBC}}=\frac{Z_{\mathrm{PBC}}^{2} e^{2}}{4 \pi \varepsilon_{0} \varepsilon} .
$$

The numerically determined electrolyte density at the Wigner-Seitz cell boundary $\rho_{\text {ws }}$ is used to calculate the screening parameter:

$$
\kappa_{\mathrm{PBC}}^{2}=e^{2} \rho_{\mathrm{WS}} / \varepsilon \varepsilon_{0} k_{B} T .
$$

The saturation of $Z_{\mathrm{PBC}}$ with increasing $Z$ has recently been confirmed experimentally [24].

Finally we consider an approximation which is empirically justified in the case of strongly interacting particles $[20,25]$. This so-called "modified DLVO approximation" (MDA) uses the DLVO potential with the renormalized charge obtained within the renormalization scheme together with a $\kappa$ calculated from $Z_{\mathrm{PBC}}$ protons plus added salt ions:

$$
U_{\mathrm{MDA}}=\frac{Z_{\mathrm{PBC}}^{2} e^{2}}{4 \pi \varepsilon_{0} \varepsilon}\left[\frac{\exp \left(\kappa_{\mathrm{MDA}} a\right)}{\left(1+\kappa_{\mathrm{MDA}} a\right)}\right]^{2}
$$


and

$$
\kappa_{\mathrm{MDA}}^{2}=e^{2}\left(2 \rho_{S}+\rho Z_{\mathrm{PBC}}\right) / \varepsilon \varepsilon_{0} k_{B} T .
$$

To demonstrate the sensitivity of the dynamical properties to the choice of the potential model we compare the predicted diffusional behavior from the fit formula (6) for these four models in Fig. 1. $D_{L} / D_{S}$ is plotted as a function of $c_{S}$ for a given set of experimental parameters. We may clearly discriminate between DLVO and MSA on the one side and PBC and MDA on the other side. The differences are most pronounced in the low salt region, where DLVO and MSA predict a lower selfdiffusion ratio than PBC or MDA. A further discrimination is not possible due to the combined errors of the analytical expression and the experimental data. We also remark that a logarithmic scale in $c_{S}$ causes a plateaulike behavior in the curves obtained from the fit formula. This does not have any physical implication, since the curves are smooth within the given relative error of $4 \%$.

It is instructive to take a more detailed look at the behavior of the analytical formula under typical experimental conditions. The dependence of the self-diffusion ratio on the true surface charge $Z$ and on the volume fraction $\Phi$ is shown for the PBC model in Figs. 2 and 3, respectively. $D_{L} / D_{S}$ is more sensitive to the packing fraction than to the true surface charge $Z$. This is due to the renormalization procedure which at high true surface charges yields very little variation in $Z_{\text {PBC }}[22-24]$. Nevertheless the diffusional behavior responds very sensitively to all three input parameters of Eq. (6). Therefore we may discriminate the four Yukawa models by accurate dynamic measurements with precisely determined experimental parameters.

The experimental data were recorded for three different volume fractions $\Phi$ between 0.00065 and 0.0041 and salt concentrations $c_{S}$ between 0 and $10^{-4} \mathrm{moll}^{-1}$. We show the comparison to the analytical formulas for the PBC and the DLVO potential models in Figs. 4 and 5 .

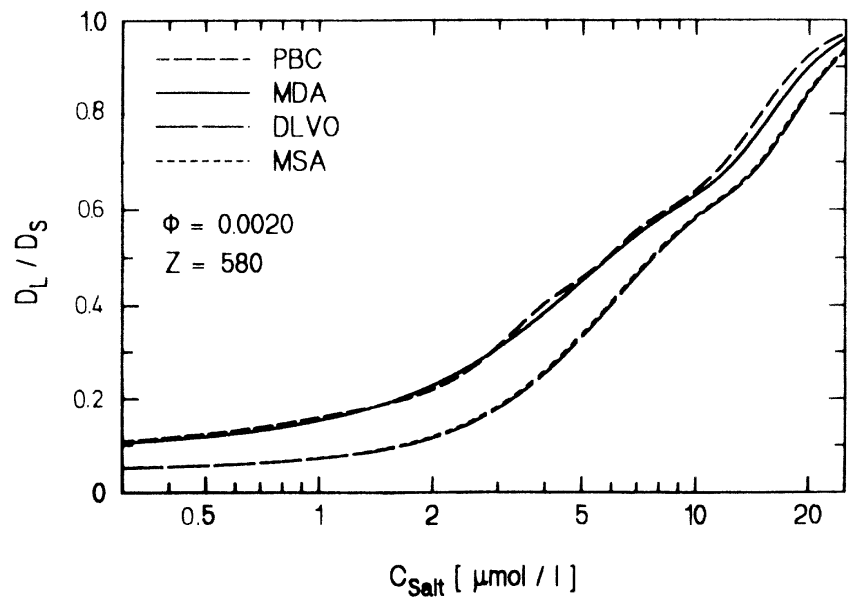

FIG. 1. Comparison of the salt concentration dependent diffusional behavior as calculated from Eq. (6) for the four Yukawa models characterized by Eqs. $(7),\left(7^{\prime}\right),\left(7^{\prime \prime}\right),\left(7^{\prime \prime \prime}\right)$ and (8), $\left(8^{\prime}\right),\left(8^{\prime \prime}\right),\left(8^{\prime \prime \prime}\right)$. Parameters: $Z=580, \Phi=0.002$.

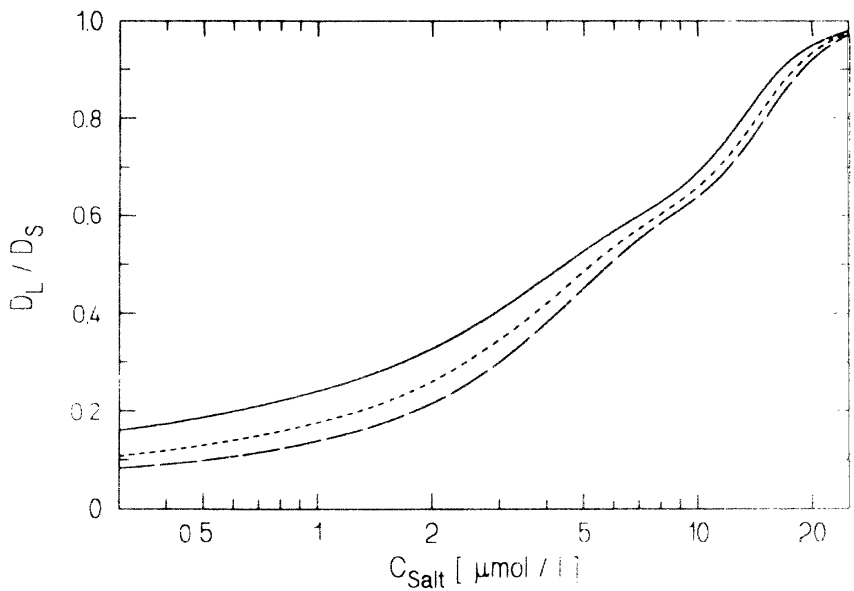

FIG. 2. Influence of the charge number on the diffusive behavior for the PBC model used with Eq. (6). Parameters: $\Phi=0.002$ and (- ): $Z=300 ;(\cdots): \quad Z=400 ;(\cdots-)$ : $Z=500$.

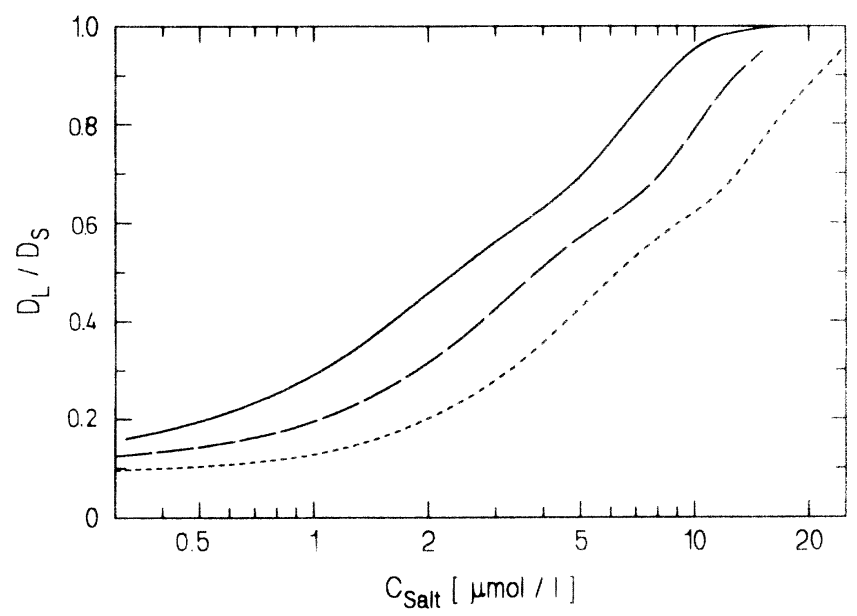

FIG. 3. Influence of the volume fraction on the diffusive behavior for the PBC model used with Eq. (6). Parameters: $Z=400$ and $(-): \Phi=0.0005 ;(--)): \Phi=0.001 ;(\cdots)$ : $\Phi=0.005$.

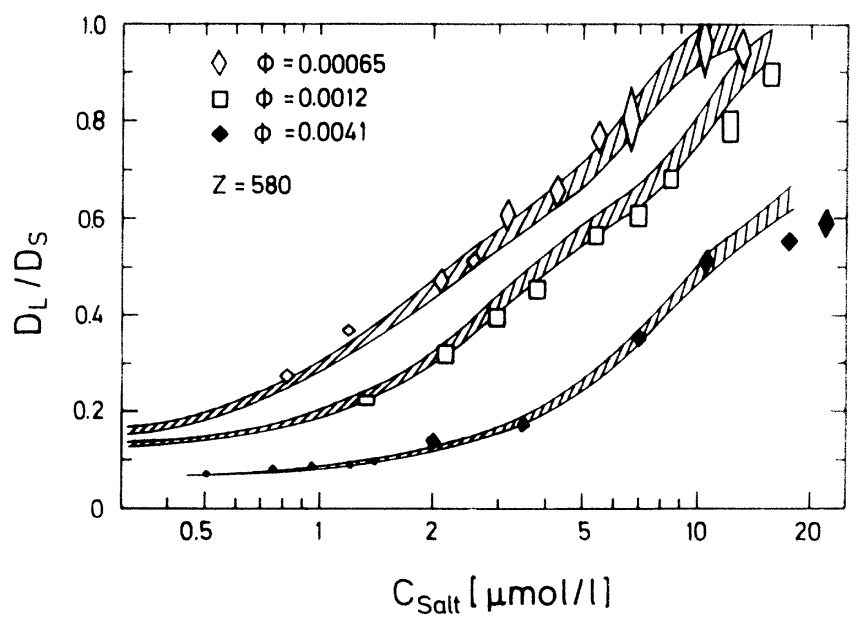

FIG. 4. Comparison of three systematic series of experimental data to Eq. (6) used with the PBC model. Experimental parameters: $Z=580$ and open diamonds: $\Phi=0.00065$; squares, $\Phi=0.0012$; filled diamonds $\Phi=0.0041$. Hatched areas: Predictions using these parameters in Eq. (6) with $4 \%$ uncertainty. 


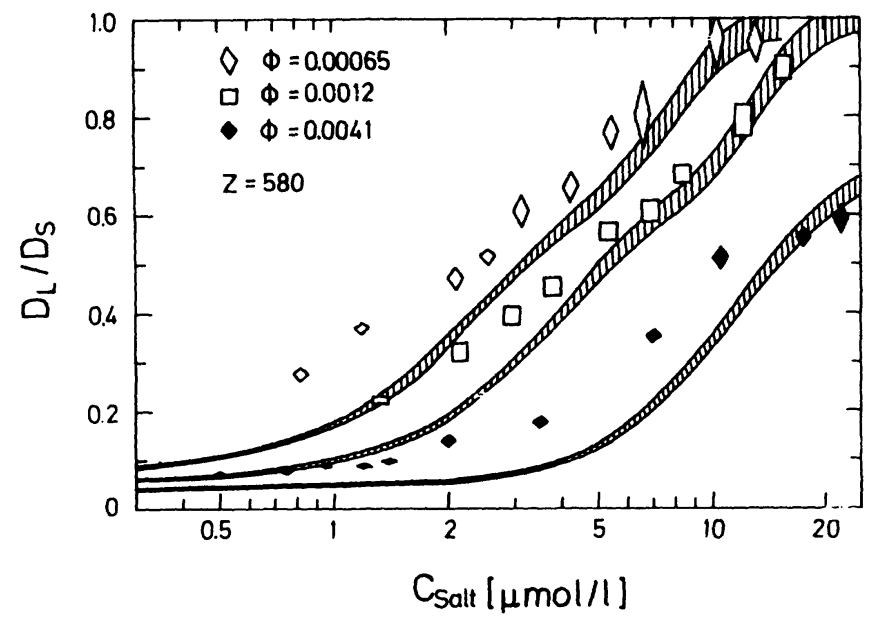

FIG. 5. As Fig. 4 but for the DLVO model.

In Fig. 4 we observe an excellent overall agreement of the predictions for the self-diffusion ratio with the FRS data in the case of the PBC model, Eqs. $\left(7^{\prime \prime}\right)$ and $\left(8^{\prime \prime}\right)$. We note that the MDA, Eqs. (7."') and (8'"), leads to equally satisfying predictions for $D_{L} / D_{S}$. Significant deviations are found in Fig. 5 for the DLVO description, Eqs. (7) and (8). These deviations are comparably smaller at low potentials but reach a factor of 2 in the low salt region. Also, the MSA, Eqs. $\left(7^{\prime}\right)$ and $\left(8^{\prime}\right)$, leads to results which are practically identical to those of the DLVO model and thus fails to describe the data.

\section{DISCUSSION}

We have performed extensive $\mathrm{BD}$ simulations and obtained a fit formula for the self-diffusion ratio $D_{L} / D_{S}$ of a Yukawa system with an accuracy of better than $4 \%$. Furthermore, our experimental system was well characterized with respect to the particle radius ( $\Delta a \leq 1 \%$ ), the true surface charge $(\Delta Z \leq 2 \%)$, the salt concentration $\left(\Delta c_{S} \leq 2 \%\right)$, and the volume fraction $(\Delta \Phi \leq 1 \%)$. On this system $D_{L}$ was measured by FRS with a statistical error of less than $1 \%$.

The achieved accuracy enables the prediction of absolute values for the self-diffusion ratio from $\mathrm{Eq}$. (6) and safe comparison to the experimental results. We note that for the first time there are no free parameters left in the description of the long-time self-diffusion of dilute but strongly interacting Yukawa systems. This allows for a clear discrimination between different models of Yukawa potentials. To appreciate the advantages of this procedure we have to compare it to other tests of the interaction potentials.

Measurements of the static structure factor $S(k)$ have been performed on similar systems [26] and a good discrimination between the PBC and the PoissonBoltzmann-Jellium model was achieved in favor of the former. Nevertheless, the particles used there were not characterized with respect to their true charge number. The renormalized charges used with the PBC model were still used as fit parameters.

The tests of different Yukawa models by comparing calculated to measured phase diagrams in principle is more sensitive. On the theoretical side both molecular dynamics simulations and perturbation theory treatments based on a Yukawa potential [Eq. (1)] have been performed [14,16,27], showing good qualitative agreement. These were compared to a number of experimentally determined phase diagrams (cf. Refs. cited in [27]) and very satisfying agreement could be achieved if the $\mathrm{Yu}$ kawa parameters were taken as fit parameters. It has been noted [1] that for systems at both low volume fractions and salt concentrations the PBC model generally yields better results than the DLVO description which performs better at high volume fractions and elevated salt concentrations. The major difficulty in performing an explicit comparison of different Yukawa models again lies within the uncertainty concerning the particle charge.

The charge dependent phase diagram of a Yukawa suspension was recorded for a system of variable but precisely measured surface group number $N$ [24]. It was shown, that a self consistent description of the phase behavior could be reached using the PBC model and Eq. (4) [14], only if the surface chemical details of the particles were accounted for. Most often, however, only the number of surface groups is known without any information about the degree of dissociation.

Only very recently we reported a technique to determine the true surface charge in situ [20]. Consequently comparisons of phase diagrams so far only had limited possibilities to discriminate between different Yukawa models, although they are much more sensible to the $\mathrm{Yu}$ kawa parameters than, for example, the static structure factor $S(k)$.

A second, more fundamental point may be raised concerning the polydispersity of the samples. Static light scattering is a method highly sensitive to contributions of polydispersity both of charge and of size $[28,4,5]$. The theoretical predictions of $S(k)$, however, are less sensitive to variations in the potential form. If large values of polydispersity are suspected, a drastic increase in the renormalized charge number is needed, in order to explain low $k$ data and the height of the first maximum in $S(k)$ [28].

In contrast to this, dynamical quantities have a high sensitivity to the potential of interaction and a much less pronounced influence of polydispersity. We have tested their dependence on both charge and size polydispersity in the BD simulations. A polydispersity of $5 \%$ in the effective charge is needed to produce significant deviations from Eq. (6).

From electrophoretic mobility measurements the charge polydispersity of our sample is estimated to be less than $5 \%$ in the number of true surface charges. The renormalized charges $Z_{\text {PBC }}$ show a much smaller variation, since their value already approaches saturation; charge polydispersity reduces to less than $1 \%$. The values for the standard deviation of the particle radii are found to be less than $2 \%$ by electron microscopy. The influence of polydispersity is therefore negligible both on the ground of the sample data and on its impact on Eq. (6). Note, however, that a change in the mean value of $Z$ by some $10 \%$ leads to a change in $D_{L}$ of more than a factor of 2 in 
the low salt region.

A final comment concerns the many-body interaction between the particles induced by nonlinear counterion screening which requires a description beyond the level of an effective pair potential as given by Eq. (1). However, based on an $a b$ initio description which explicitly treats the counterions [29], it was recently shown [30] that the many-body forces can satisfactorily be fitted by an optimal pair potential whose shape is very close to the $\mathrm{Yu}$ kawa form. Unfortunately there is no simple analytical formula for the corresponding Yukawa parameters, in contrast to the models discussed in Sec. IV. Still, once these parameters are known, our fit formula provides a simple, direct determination of $D_{L} / D_{S}$.

In conclusion, the validity of the PBC model has been verified with high accuracy absolute measurements of a dynamical quantity and without any free parameters. This is a major extension as compared to former tests. Note, however, that the MDA also provides an excellent description of the data. Belloni gives a value for the maximum renormalized charge number as $Z_{\mathrm{PBC}}$ $\leq 4 a(1+\kappa a) / L_{B}$, where $L_{B}=e^{2} / 4 \pi \varepsilon \varepsilon_{0} k_{B} T$ is the Bjerrum length [31]. Saturation qualitatively consistent with this prediction was recently verified on a system of ionicnonionic mixed micelles with a maximum structural charge of 75 [32] from fits to static neutron scattering data. In the case of highly charged colloidal spheres and of the renormalized charge approaching saturation, the MDA together with our fit formula then provides a quick estimation procedure for the self-diffusion ratio.

\section{ACKNOWLEDGMENTS}

The authors would like to thank W. Härtl for providing the PBC program. We thank the referee for guiding our attention to Ref. [32]. Financial support of the Deutsche Forschungs Gemeinschaft (SFB 306) is gratefully acknowledged.
[1] P. N. Pusey, in Liquids, Freezing, and Glass Transitions, 51st Les Houches Summer School, 1989, edited by J. P. Hansen, D. Levesque, and J. Zinn-Justin (Elsevier, Amsterdam, 1991), p. 763.

[2] T. G. M. van de Veen, Colloidal Hydrodynamics (Academic, London, 1989).

[3] R. J. Hunter, Foundations and Colloidal Science (Oxford University Press, New York, 1989).

[4] H. Löwen, Phys. Rep. 237, 249 (1994).

[5] R. Klein, in Structure and Dynamics of Strongly Interacting Colloids and Supramolecular Aggregates, Vol. 369 of NATO Advanced Study Institute, edited by S.-H. Chen, J. S. Huang, and P. Tartaglia (Kluwer, Dordrecht, 1992), p. 39.

[6] D. N. Petsev and N. D. Denkov, J. Colloid Interface Sci. 149, 329 (1992).

[7] W. Härtl, H. Versmold, U. Wittig, and P. Linse, J. Chem. Phys. 97, 7797 (1992).

[8] M. M. Kops-Werkhoven and H. M. Fijnaut, J. Chem. Phys. 77, 2242 (1988); M. M. Kops-Werkhoven, C. Pathmamanoharan, A. Vrij, and H. M. Fijnaut, ibid. 77, 5913 (1988); R. H. Ottewill and N. St. J. Williams, Nature (London) 325, 232 (1987); A. van Veluwen and H. N. W. Lekkerkerker, Phys. Rev. A 38, 3758 (1988); W. van Megen and S. M. Underwood, J. Chem. Phys. 91, 552 (1989); V. Degiorgio, R. Piazza, M. Corti, and J. Stavans, J. Chem. Soc. Faraday Trans. 87, 431 (1991).

[9] W. D. Dozier, H. M. Lindsay, and P. M. Chaikin, J. Phys. (Paris) Colloq. 46, C3-165 (1985).

[10] H. Löwen, T. Palberg, and R. Simon, Phys. Rev. Lett. 70, 1557 (1993).

[11] R. Simon, T. Palberg, and P. Leiderer, J. Chem. Phys. 99, 3030 (1993).

[12] A. van Blaaderen, J. Peetermanns, J. Maret, and J. K. G. Dhont, J. Chem. Phys. 96, 4591 (1992).

[13] H. Löwen and G. Szamel, J. Phys. Condens. Matter 5, 2295 (1993).

[14] M. O. Robbins, K. Kremer, and G. S. Grest, J. Chem.
Phys. 88, 3286 (1988).

[15] H. Löwen, J. P. Hansen, and J. N. Roux, Phys. Rev. A 44, 1169 (1991); D. L. Ermak, J. Chem. Phys. 62, 4189 (1975); 62, 4197 (1975).

[16] E. J. Meijer and D. Frenkel, J. Chem. Phys. 94, 2269 (1991).

[17] M. J. Stevens and M. O. Robbins, J. Chem. Phys. 98, 2319 (1993).

[18] N. Pistoor and K. Kremer, Physica A 201, 171 (1993).

[19] T. Palberg, W. Härtl, U. Wittig, H. Versmold, M. Würth, and E. Simnacher, J. Phys. Chem. 96, 8180 (1992).

[20] T. Palberg, J. Kottal, F. Bitzer, R. Simon, M. Würth, and P. Leiderer, J. Colloid. Interface Sci. (to be published).

[21] L. Belloni, J. Chem. Phys. 85, 519 (1986).

[22] L. Belloni, M. Drifford, and P. Turq, Chem. Phys. 83, 147 (1984).

[23] S. Alexander, P. M. Chaikin, P. Grant, G. J. Morales, P. Pincus, and D. Hone, J. Chem. Phys. 80, 5776 (1984).

[24] T. Palberg, W. Mönch, F. Bitzer, P. Leiderer, L. Belloni, T. Bellini, and R. Piazza, Helv. Phys. Acta (to be published).

[25] W. D. Dozier, H. M. Lindsay, and P. M. Chaikin, J. Phys. (Paris) Colloq. 46, C3-257 (1985).

[26] W. Härtl and H. Versmold, J. Chem. Phys. 88, 7157 (1988).

[27] L. P. Voegtli and Ch. F. Zukoski IV, J. Colloid Interface Sci. 141, 79 (1991).

[28] B. D'Aguanno, R. Krause, J. N. Mendez-Alcarez, and R. Klein, J. Phys. Condens. Matter 4, 3077 (1992).

[29] H. Löwen, J. P. Hansen, and P. A. Madden, Phys. Rev. Lett. 68, 1081 (1992); J. Chem. Phys. 98, 3275 (1993).

[30] H. Löwen and G. Kramposthuber, Europhys. Lett. 23, 673 (1993).

[31] L. Belloni, Chem. Phys. 99, 43 (1985), and private communication.

[32] S. Bucci, C. Fagotti, V. Degiorgio, and R. Piazza, Langmuir 7, 824 (1991). 